\title{
Efeito musculoesquelético do exercício resistido em idosos: revisão sistemática
}

\section{Musculo-skeletal effects of resistance exercises in the elderly}

Leandro Augusto Menezes Rego ${ }^{1}$. Marcelo de Barros Patriota Filhoํ․ Júlio Cesar Chagas e Cavalcante ${ }^{2}$. João Paulo Tavares Linhares ${ }^{3}$. José Alberto dias Leite ${ }^{4}$.

1 Residente em Ortopedia e Traumatologia, Universidade Federal do Ceará, Fortaleza, Ceará, Brasil. 2 Mestre em Cirurgia, Preceptor no setor de Traumato-Ortopedia/Anatomia Humana/Internato, Universidade Federal do Ceará, Fortaleza, Ceará, Brasil. 3 Especialista em Ortopedia e Traumatologia, Preceptor no serviço de Formação e Treinamento em Traumato-Ortopedia, Universidade Federal do Ceará, Fortaleza, Ceará, Brasil. 4 Doutor em Medicina, professor titular do Departamento de Cirurgia da Universidade Federal do Ceará (UFC), Membro da Coordenação da Pós-Graduação em Cirurgia, Fortaleza, Ceará, Brasil.

\section{RESUMO}

Considera-se exercício resistido uma atividade física que envolva um estímulo muscular para deslocar certa quantidade de massa, acelerando-a a partir do repouso e englobando um ou mais grupos musculares. O processo degenerativo das fibras musculares está implicado na gênese da vulnerabilidade e da perda funcional. A prática regular de exercício resistido, talvez, possa reverter, em parte, essa perda funcional. Esta revisão de literatura teve como base de dados os artigos contidos na PubMed, que foram coletados randomicamente a partir dos seguintes descritores: terapia por exercício, envelhecimento, desenvolvimento muscular, sarcopenia, dinapenia e síndrome de fragilidade. Foram selecionados 19 artigos a partir da base de dados da PubMed. Dentre eles, têm-se: 9 ensaios clínicos randomizados, quatro estudos com grupo controle, duas meta-análises, um estudo longitudinal com intervenção sem grupo controle, dois de revisão e um consenso internacional. Acreditamos que os temas sobre os quais discorremos ainda carecem de mais evidências experimentais. Contudo, apresentam um saldo positivo de evidências favoráveis à prática do exercício resistido em idosos.

Palavras-chave: Terapia por exercício. Envelhecimento. Desenvolvimento muscular. Sarcopenia. Dinapenia. Síndrome de Fragilidade.

\section{ABSTRACT}

Resistance exercise is considered a physical activity composed by muscular stimulus capable of moving certain quantity of mass, which is accelerated from rest and evolves one or more muscular groups. Muscular fiber's degenerative process is involved in the genesis of vulnerability and functional loss. Regular practice of resisted exercise might partly reverse this functional loss. This literature review was made from PubMed articles database, which was collected randomly according to the following keywords: exercise therapy, aging, muscle development, sarcopenia, dynapenia and frailty syndrome. There are among these articles: 9 randomized clinical trials, four control group studies, two meta-analyses, one longitudinal study with intervention and without control group, 2 review studies and one international consensus. We strongly believe that the themes we studied still miss a bigger number of experimental evidences; however, they also present a positive balance of evidences that is favorable for the practice of resistance exercise by seniors.

Keywords: Exercise therapy. Aging. Muscle development. Sarcopenia. Dynapenia. Frailty Syndrome.

Autor correspondente: Leandro Augusto Menezes Rêgo, Rua Coronel Linhares, 2220, apartamento 802, Dionísio Torres, Fortaleza, Ceará. CEP: 60170-241.Telefone: +55 85 99973-0523. E-mail: leandrorego@gmail.com

Conflito de interesses: Não há qualquer conflito de interesses por parte de qualquer um dos autores.

Recebido em: 08 Abril 2016; Revisado em: 20 Jul 2016; Aceito em: 21 Jul 2016. 


\section{INTRODUÇÃO}

Com quedas progressivas nas taxas de mortalidade e principalmente das de fecundidade, estão lançadas as bases demográficas para o envelhecimento de uma população. Em alguns países, esses eventos ocorreram de maneira gradual, de modo que essas sociedades tiveram oportunidade de desenvolver algum tipo de estratégia de enfrentamento diante das demandas de uma população mais velha. Outros países, grupo em que se inclui o Brasil, enfrentaram uma redução mais rápida desses indicadores e não tiveram a mesma oportunidade para desenvolver as referidas estratégias.

O envelhecimento, por sua vez, é fator de risco para o desenvolvimento de situações como a cascata degenerativa das fibras musculares, que está implicada na gênese da vulnerabilidade e da perda funcional, podendo ser associada a vários processos patológicos. ${ }^{1}$ Dentre tais processos, podemos citar o aumento do tecido adiposo intermuscular que leva a uma maior comorbidade, uma vez que aumenta a probabilidade de internações hospitalares, de invalidez (dificuldade de deambular, deficit de força muscular, limitação do potencial para aerobiose muscular, etc) e aumento da resistência insulínica periférica; ${ }^{2}$ a síndrome metabólica, que está associada a um maior risco cardiovascular (aterosclerose), a obesidade, a hipertensão arterial sistêmica e a dislipidemia; a osteoporose (elevado risco para fraturas) e a sarcopenia (alterações na marcha, desequilíbrio, quedas e fraturas). ${ }^{3}$

Em 2010, o European Working Group on Sarcopenia in Older People (EWGSOP), no intuito de promover uma melhor identificação e tratamento da sarcopenia, sugere, em consenso, que a mesma seja reconhecida como uma síndrome geriátrica. Sendo a sarcopenia caracterizada pela perda progressiva e generalizada de massa muscular esquelética e força associadas ao risco de resultados adversos tais como incapacidade, baixa qualidade de vida e morte, o EWGSOP recomenda o diagnóstico baseado na redução da massa muscular, obrigatoriamente associada à redução da força muscular e do desempenho físico. ${ }^{4}$

A prática regular de exercício resistido está relacionada com uma melhora no controle das entidades patológicas supracitadas, bem como daquelas eminentemente relacionadas à senilidade e à resposta inflamatória, talvez, por um melhor controle da ativação de fatores imunológicos antiinflamatórios. ${ }^{5}$ Ressalte-se o fato de que esse tipo de exercício estaria relacionado a uma redução da perda de matriz óssea e a uma diminuição no processo de atrofia senil muscular e, assim, diminuiria a incidência e a prevalência de quedas em idosos, decorrentes da sarcopenia e da osteoporose (sítios comuns de fratura pós-queda: vértebras, quadril, rádio distal; cuja incidência aumenta a partir dos 60 anos). ${ }^{6}$

Considera-se exercício resistido uma atividade física que envolva um estímulo muscular para deslocar certa quantidade de massa, acelerando-a a partir do repouso e englobando um ou mais grupos musculares. ${ }^{3}$ No tocante a esse intento, alguns fatos deveriam ser observados com cautela ao empregar- se essa modalidade de atividade a indivíduos idosos no intuito de promover um trabalho de ganho de força muscular e incremento de matriz óssea: 1) a resposta depende do trabalho de cada grupo muscular; 2) a carga a ser abarcada pelo sistema osteomuscular deve ser maior que aquela habitualmente suportada a fim de que a resposta trófica ocorra de maneira diretamente proporcional ao aumento de carga; 3) a remineralização óssea é tão duradoura quanto o estímulo trófico; 4) os melhores resultados serão vistos nos indivíduos mais osteopênicos/osteoporóticos; 5) todos tem um limite de resposta a uma atividade física e, eventualmente, atingirão o seu máximo. ${ }^{6}$

O presente trabalho tem como objetivo estabelecer as evidências mais recentes a partir de uma revisão de literatura acerca dos benefícios do exercício resistido em indivíduos idosos, bem como a sua relação com a melhora da qualidade do envelhecimento dessas pessoas e sua repercussão na saúde óssea e muscular das mesmas, discutindo as suas implicações nas entidades patológicas supracitadas.

\section{MÉTODOS E MATERIAIS}

Esta revisão de literatura teve como base de dados os artigos contidos na PubMed, que foram coletados randomicamente a partir das seguintes palavras-chave: terapia por exercício, envelhecimento, desenvolvimento muscular e sarcopenia. Para a confecção deste trabalho, uma pergunta serviu de ponto de partida: "O exercício físico resistido teria algum efeito terapêutico no desenvolvimento muscular de indivíduos idosos com sarcopenia?". O levantamento da literatura considerou trabalhos de revisão, ensaios clínicos randomizados, grupos controle, metanálises e consensos. Os descritores foram obtidos a partir da Biblioteca Virtual em Saúde (BIREME). A busca foi realizada em língua inglesa (PubMed). Foram incluídos, posteriormente, trabalhos sobre o exercício resistido por causa de sua grande relevância em termos de evidências até o momento. Nos demais trabalhos a serem incluídos, também se considerou o grau de relevância de suas evidências (data de publicação até 1999, estudos com $\mathrm{n}^{\mathrm{o}}$ de pelo menos 10 indivíduos, horizontalidade do estudo, uso de metodologias similares, conflitos de interesse), logo, aqueles que não possuíam tais requisitos foram excluídos. Não se pode quantificar grau de significância dos diversos trabalhos numericamente. O estudo pretende analisar os idosos de uma forma geral, visando a avaliar a senilidade e sua eventual sarcopenia de modo mais amplo.

\section{RESULTADOS}

Foram selecionados 19 artigos a partir da base de dados da PubMed. Dentre eles, têm-se: 9 ensaios clínicos randomizados ${ }^{2,7-14}$ (sendo dois ensaios duplo-cego ${ }^{8,14}$, cinco com grupo controle); quatro estudos com grupo controle; duas meta-análises; um estudo longitudinal com intervenção sem grupo controle, dois de revisão e um consenso internacional. A construção deste trabalho contou apenas com critérios de 
inclusão em virtude da escassez de trabalhos que abordassem especificamente o tema: "Efeito musculoesquelético do exercício resistido em idosos". Logo, não foram atribuídos critérios de exclusão para a busca randomizada de trabalhos realizada.

Os trabalhos apresentaram alguns pontos de intersecção com avaliações para determinar que modalidade de exercícios melhoraria a capacidade de equilíbrio nos idosos, bem como se haveria importância na frequência com que os indivíduos estudados se exercitariam. Outro parâmetro em comum seria a densidade mineral óssea e a massa magra muscular. $\mathrm{O}$ ganho de força através do exercício resistido seria outro aspecto bastante mencionado.

Sendo mais objetivo, os ensaios clínicos estudados tentaram avaliar: se haveria relação entre a frequência com que idosos realizariam exercício e a densidade mineral óssea; exercício físico resistido e exercício tradicional de equilíbrio, se um ou outro seria melhor para aprimorar a capacidade de equilíbrio de indivíduos idosos; se a associação entre exercício físico e dieta hipocalórica ofereceria algum implemento no processo de perda de peso em idosos obesos; se o uso de acetominofeno afetaria a síntese protéica no tecido muscular e ósseo, interferindo no mecanismo de adaptação ao exercício resistido progressivo; se haveria alguma diferença significativa na capacidade de redução de tecido adiposo intermuscular através do exercício físico resistido, bem como uma possível diferença entre a forma de execução dessa modalidade de exercício físico tradicional e excêntrica; se o exercício resistido de moderada intensidade, em mulheres com diagnóstico de osteoartrite no joelho póstratamento de câncer mamário, manteria a massa muscular e a densidade óssea; se haveria implemento cognitivo e/ou físico à mulheres idosas submetidas a uma série de exercícios físicos variados; os possíveis efeitos da perda de peso e do exercício físico em idosos. Trabalhos com grupos controles buscaram: relacionar exercício de equilíbrio e exercício resistido em relação à marcha e a uma provável redução de quedas; avaliar idosos com pequenas incapacidades funcionais submetidos a séries variadas de exercícios por 12 meses quanto à performance física per si, performance física diária funcional e qualidade de vida; verificar a resposta imune musculoesquelética em pacientes submetidos a um exercício agudo em comparação àqueles submetidos ao exercício resistido; avaliar jovens voluntários submetidos a exercício físico resistido quanto a tarefas que exijam um bom controle motor. Já as metanálises, tentaram uma determinação dos efeitos do exercício resistido sobre a massa magra muscular e a efetividade do exercício resistido em idosos para ganho de força muscular. De maneira peculiar e oportuna, um estudo longitudinal tentou avaliar se a ingestão de aminoácidos essenciais sinalizaria para o início da síntese protéica muscular em jovens e idosos de maneira similar. Os trabalhos de revisão tiverem um intento semelhante ao deste presente trabalho e discutiram acerca das temáticas já expostas.

Abaixo apresentamos quadros-resumo comparativos (Quadros 1 e 2) com informações sobre os artigos incluídos na revisão, visando a determinar suas especificidades.

Quadro 1. Comparativo moldes/populações

\begin{tabular}{|c|c|c|c|c|c|}
\hline $\begin{array}{l}\text { Detalhes do } \\
\text { Estudo }\end{array}$ & \multicolumn{2}{|c|}{ Moldes do Estudo } & \multicolumn{3}{|c|}{ População estudada } \\
\hline Autor, ano & Tipo & $\begin{array}{c}\text { Número de } \\
\text { estudos }\end{array}$ & $\mathrm{n}$ & Características & Idade (anos) \\
\hline Peterson, 2010 & Meta análise & 47 & $\begin{array}{c}1079 \\
(584 \mathrm{H}: 495 \mathrm{M}) \\
\end{array}$ & $\begin{array}{l}\text { Homens e mulheres idosos, residentes em instituições, } \\
\text { hospitais ou comunidades de idosos }\end{array}$ & $67,4 \pm 6,3$ \\
\hline Jacobs, 2014 & Ensaio Clínico & 1 & 77 (20H:57M) & Idosos com múltiplas comorbidades e história de quedas & $75,5 \pm 6,8$ \\
\hline Sundell, 2011 & Artigo de Revisão & 45 & & & \\
\hline $\begin{array}{l}\text { Cruz-Jentoft, } \\
2010\end{array}$ & Consenso & 75 & & $\begin{array}{l}\text { Os parâmetros escolhidos para estudo nas populações foram: } \\
\text { reduções em massa muscular (índice de massa esquelética), } \\
\text { força muscular (força preênsil) e performance física (cadência } \\
\text { dissociada ou não da Bateria de Performance Física Curta } \\
\text { [equilíbrio, marcha, força e resistência]). }\end{array}$ & \\
\hline Gordon, 2011 & $\begin{array}{l}\text { Análise de } \\
\text { microarranjo }\end{array}$ & & $7(4 \mathrm{H}: 3 \mathrm{M})$ & $\begin{array}{l}\text { Idade entre } 18 \text { e } 40 \text { anos; ausência de doenças crônicas; ausência } \\
\text { de história prévia de exercícios resistidos; não consumir } \\
\text { medicações ou suplementos que afetem a musculatura }\end{array}$ & $18>i<40$ \\
\hline Rutheford, 1999 & Revisão de literatura & 66 & & & \\
\hline Ashe, 2012 & $\begin{array}{l}\text { Ensaio Clínico } \\
\text { (Randomizado) }\end{array}$ & 37 & $\begin{array}{c}\text { 147M (100 } \\
\text { concluíram) }\end{array}$ & \begin{tabular}{|llll}
$\begin{array}{l}\text { Mulheres pós-menopausa, saudáveis, residentes } \\
\text { comunidade }\end{array}$ & na \\
\end{tabular} & $65-75$ \\
\hline Lange, 2009 & $\begin{array}{l}\text { Ensaio Clínico } \\
\text { (Randomizado) }\end{array}$ & 57 & & $\begin{array}{l}\text { Mulheres }>40 \text { anos, saudáveis, com osteoartrite primária em, } \\
\text { pelo menos, um joelho. }\end{array}$ & $>40$ \\
\hline $\begin{array}{c}\text { Winters-Stone, } \\
2011\end{array}$ & $\begin{array}{l}\text { Ensaio Clínico } \\
\text { (Randomizado) }\end{array}$ & 64 & $106 \mathrm{M}$ & $\begin{array}{l}\text { Mulheres com câncer de mama em estágio inicial, a mais } \\
\text { de um ano pós-radioterapia e/ou quimioterapia, } \geq 50 \text { anos no } \\
\text { diagnóstico ou pós-menopausa, sem osteoporose ou sem usar } \\
\text { medicações para perda óssea, vírgens de exercícios de impacto } \\
\text { ou resistidos e autorizadas por um médico do esporte. }\end{array}$ & $>50$ \\
\hline
\end{tabular}

Continua 
Conclusão

\begin{tabular}{|c|c|c|c|c|c|}
\hline $\begin{array}{l}\text { Detalhes do } \\
\text { Estudo }\end{array}$ & \multicolumn{2}{|c|}{ Moldes do Estudo } & \multicolumn{3}{|c|}{ População estudada } \\
\hline Autor, ano & Tipo & \begin{tabular}{|c|}
$\begin{array}{c}\text { Número de } \\
\text { estudos }\end{array}$ \\
\end{tabular} & $\mathrm{n}$ & Características & Idade (anos) \\
\hline Joshua, 2014 & $\begin{array}{l}\text { Ensaio Clínico } \\
\text { (Randomizado) }\end{array}$ & 30 & 54 & $\begin{array}{l}\text { Foram avaliados idosos moradores de } 4 \text { lares de idosos, com } \\
\text { mais de } 65 \text { anos em Mangalore, sul da Índia. } \\
\text { Foram excluídos do estudo idosos que apresentavam doenças } \\
\text { cardiovasculares sintomáticas, doenças neurológicas, } \\
\text { neuropatias periféricas dos membros inferiores com } \\
\text { perda sensitiva significativa da coluna dorsal, doenças } \\
\text { musculoesqueléticas no quartil inferior que pudessem interferir } \\
\text { com os resultados, malignidades, medicações que possuem } \\
\text { risco para quedas, distúrbios vestibulares diagnosticados e } \\
\text { pacientes que foram submetidos a treinamento de força para } \\
\text { membros inferiores e/ou equilíbrio nos últimos } 3 \text { meses. }\end{array}$ & 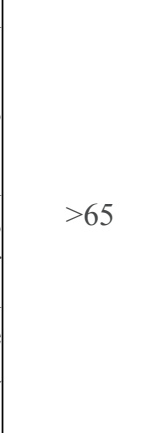 \\
\hline Vaughan, 2012 & $\begin{array}{l}\text { Ensaio Clínico } \\
\text { (Randomizado) }\end{array}$ & 56 & $100 \mathrm{M}$ & $\begin{array}{l}\text { Mulheres com idade entre } 65 \text { e } 75 \text { anos, sem alteração } \\
\text { cognitiva, que realizavam menos de } 60 \text { minutos de atividade } \\
\text { física por semana, com disponibilidade para frequentar aulas, } \\
\text { duas vezes por semana, em dias da semana. }\end{array}$ & $65-75$ \\
\hline Frimel, 2008 & $\begin{array}{l}\text { Ensaio Clínico } \\
\text { (Randomizado) }\end{array}$ & 38 & 30 (18M:12H) & $\begin{array}{l}\text { Idosos obesos submetidos a dieta/terapia comportamental }(\mathrm{n}= \\
\text { 15) ou dieta/terapia comportamental associadas a exercícios } \\
\text { de resistência progressiva. }(\mathrm{n}=15) \text {. }\end{array}$ & $70 \pm 5$ \\
\hline Villareal, 2011 & $\begin{array}{l}\text { Ensaio Clínico } \\
\text { (Randomizado) }\end{array}$ & 40 & $\begin{array}{c}107(93 \\
\text { concluíram) }\end{array}$ & $\begin{array}{l}\text { Idosos obesos sedentários, com peso mantido no último ano e } \\
\text { sem alteração das medicações nos últimos seis meses. }\end{array}$ & $>65$ \\
\hline $\begin{array}{l}\text { Jankowski, } \\
2012\end{array}$ & $\begin{array}{l}\text { Ensaio Clínico } \\
\text { (Randomizado) }\end{array}$ & 33 & $\begin{array}{c}26 \mathrm{H}(23 \\
\text { concluíram })\end{array}$ & $\begin{array}{l}\text { Homens na faixa etária estudada que utilizaram acetomifeno } \\
\text { ou antinflamatórios não esteroidais por menos de três dias } \\
\text { por mês. Foram excluídos os voluntários que realizavam } \\
\text { exercícios regulares. }\end{array}$ & $>50$ \\
\hline Carroll, 2001 & Ensaio Clínico & 25 & $16(8 \mathrm{M}: 8 \mathrm{H})$ & Adultos jovens saudáveis. & $18-32$ \\
\hline Kim, 2010 & $\begin{array}{l}\text { Ensaio Clínico } \\
\text { (Randomizado) }\end{array}$ & 28 & 18 & Idosos voluntários recrutados sem limitações físicas. & $>65$ \\
\hline Taguchi, 2010 & Ensaio Clínico & 42 & 65 (53M:12H) & Idosos provenientes de casas de repouso. & $>70$ \\
\hline Peterson, 2011 & Revisão de literatura & 108 & & & \\
\hline $\begin{array}{l}\text { Drummond, } \\
2008\end{array}$ & Ensaio Clínico & 62 & $13 \mathrm{H}$ & $\begin{array}{l}\text { Homens adultos jovens e idosos sedentários e fisicamente } \\
\text { saudáveis. }\end{array}$ & $24-77$ \\
\hline
\end{tabular}

Fonte: elaborado pelos autores.

Quadro 2. Comparativo Viés/resultados.

\begin{tabular}{|c|c|c|c|}
\hline $\begin{array}{c}\begin{array}{c}\text { Detalhes do } \\
\text { estudo }\end{array} \\
\end{array}$ & Detalhes da intervenção & \multirow{2}{*}{ Fragilidade/viés do estudo } & \multirow{2}{*}{ Resultados } \\
\hline Autor, ano & Duração (semanas) & & \\
\hline Peterson, 2010 & $\begin{array}{c}6-52 \\
(17,6 \pm 8,6)\end{array}$ & Heterogeneidade entre os estudos & $\begin{array}{l}\text { Exercícios resistidos são efetivos para melhora } \\
\text { da força entre idosos, particularmente com } \\
\text { treinamentos de maior intensidade }\end{array}$ \\
\hline Jacobs, 2014 & 12 & $\begin{array}{l}\text { Todos os pacientes apresentavam múltiplas } \\
\text { comorbidades (de base) associadas a história } \\
\text { de quedas, então os resultados não podem ser } \\
\text { generalizados para a população de idosos "não } \\
\text { desabilitados"; } \\
\text { Amostragem relativamente pequena quando } \\
\text { separada por grupos; } \\
\begin{array}{l}\text { Ausência de grupo inativo (controle) para } \\
\text { comparação de resultados }\end{array}\end{array}$ & $\begin{array}{l}\text { Participantes no grupo tradicional apresentaram } \\
\text { perda significativa de massa magra nos nove } \\
\text { meses após intervenção, enquanto participantes } \\
\text { no grupo excêntrico não. Quando houve distinção } \\
\text { entre grupos com altos e baixos níveis de tecido } \\
\text { adiposo intermuscular, houve respostas diferentes, } \\
\text { com o grupo intervenção apresentando redução } \\
\text { dos níveis de Tecido Adiposo Intramuscular } \\
(\text { TAIM). }\end{array}$ \\
\hline
\end{tabular}


Continuação

\begin{tabular}{|c|c|c|c|}
\hline $\begin{array}{l}\text { Detalhes do } \\
\text { estudo }\end{array}$ & Detalhes da intervenção & \multirow{2}{*}{ Fragilidade/viés do estudo } & \multirow{2}{*}{ Resultados } \\
\hline Autor, ano & Duração (semanas) & & \\
\hline Sundell, 2011 & & & $\begin{array}{l}\text { Exercício resistido é, provavelmente, a medida } \\
\text { mais efetiva para prevenir e tratar a sarcopenia. } \\
\text { Além disso, vários estudos demostraram que o } \\
\text { exercício resistido pode manter ou aumentar a } \\
\text { Densidade Mineral Óssea (DMO). }\end{array}$ \\
\hline $\begin{array}{l}\text { Cruz-Jentoft, } \\
2010\end{array}$ & & $\begin{array}{l}\text { Compila um conjunto de ferramentas que auxiliam } \\
\text { na promoção do reconhecimento e tratamento } \\
\text { da sarcopenia relacionada ao envelhecimento } \\
\text { apenas, mas não relacionada às outras causas. }\end{array}$ & $\begin{array}{l}\text { Ofereceumadefiniçãopráticadasarcopenia, sumariza } \\
\text { o conhecimento atual e mecanismos subjacentes } \\
\text { e revisa técnicas para mensuração das variáveis } \\
\text { associadas à sarcopenia. Oferece ainda parâmetros } \\
\text { para uso dessas ferramentas na identificação e } \\
\text { avaliação da efetividade do tratamento. }\end{array}$ \\
\hline Gordon, 2011 & 12 & $\begin{array}{l}\mathrm{N}^{\circ} \text { de pacientes muito reduzido; idade da } \\
\text { população estudada fora da idade alvo da revisão; }\end{array}$ & \\
\hline Rutheford, 1999 & & & $\begin{array}{l}\text { Ainda que não esteja definida a melhor ou menor } \\
\text { intensidade de exercícios para alcançar a redução } \\
\text { ou reversão na perda óssea relacionada ao } \\
\text { envelhecimento, dados epidemiológicos indicam } \\
\text { que uma história de atividades físicas podem reduzir } \\
\text { a incidência de fraturas, particularmente no quadril. }\end{array}$ \\
\hline Ashe, 2012 & 52 & $\begin{array}{l}\text { 1) As participantes do estudo eram bastante ativas } \\
\text { e, portanto, podem não representar a população } \\
\text { idosa geral enquanto limitam a generalização dos } \\
\text { resultados para subgrupos de idosas ativas. } \\
\text { 2) A obtenção de resultados de medidas ósseas por } \\
\text { Peripheral Quantitative Computed Tomography } \\
\text { (pQCT) ocorre em sítios periféricos e não } \\
\text { caracteriza compartimentos ósseos clinicamente } \\
\text { relevantes como o fêmur proximal. }\end{array}$ & $\begin{array}{l}\text { Idosas fisicamente ativas tem a capacidade de } \\
\text { manter a densidade cortical, área total e força } \\
\text { óssea tibial ao longo de } 1 \text { ano. Por outro lado, o } \\
\text { regime ideal para promover esse benefício ainda } \\
\text { não está claro. }\end{array}$ \\
\hline Lange, 2009 & 26 & & \\
\hline $\begin{array}{c}\text { Winters-Stone, } \\
2011\end{array}$ & 52 & $\begin{array}{l}\text { 1) O grupo estudado que é composto por mulheres } \\
\text { com câncer de mama, que mesmo em estágios } \\
\text { iniciais, não compõe a média da população; } \\
\text { 2) Ausência de um grupo controle; } \\
\text { 3) Inclusão de participantes aerobicamente ativas. }\end{array}$ & $\begin{array}{l}\text { O programa POWIR proposto pelos autores } \\
\text { demostrou resultados no sentido de preservação } \\
\text { óssea e lentificação do turnover e ambos } \\
\text { contribuem para reduzir o risco de fraturas da } \\
\text { coluna. }\end{array}$ \\
\hline Joshua, 2014 & 26 & & $\begin{array}{l}\text { De modo geral, todos os pacientes demonstraram } \\
\text { melhora do equilíbrio, sendo o subgrupo do } \\
\text { exercício resistido progressivo o que apresentou } \\
\text { melhores resultados. }\end{array}$ \\
\hline Vaughan, 2012 & 16 & $\begin{array}{l}\text { 1) O estudo avaliou apenas indivíduos do sexo } \\
\text { feminino; } \\
\text { 2) Não foi estabelecida relação entre as variáveis } \\
\text { avaliadas. }\end{array}$ & $\begin{array}{l}\text { O estudo conclui que devem ser acrescentadas } \\
\text { variáveis mais específicas para determinar } \\
\text { a relação entre exercício multimodal, idade, } \\
\text { cognição e neurotrofinas. }\end{array}$ \\
\hline Frimel, 2008 & 24 & & $\begin{array}{l}\text { A adição de exercício a um programa de reeducação } \\
\text { alimentar reduz a perda de massa muscular durante } \\
\text { perda ponderal voluntária em adultos obesos e } \\
\text { significativamente aumenta sua resistência física. }\end{array}$ \\
\hline Villareal, 2011 & 52 & & $\begin{array}{l}\text { Os achados sugerem que perda ponderal ou } \\
\text { exercício isolados promovem melhoria na } \\
\text { condição física e redução da fragilidade de adultos } \\
\text { obesos idosos. }\end{array}$ \\
\hline $\begin{array}{l}\text { Jankowski, } \\
2012\end{array}$ & 16 & $\begin{array}{l}\text { O estudo visa avaliar a associação entre uma } \\
\text { medicação e o exercício resistido. }\end{array}$ & \begin{tabular}{|l} 
O uso diário de $1 \mathrm{~g}$ de acetomifeno não \\
comprometeu o aumento de massa muscular \\
durante o período de exercício resistido.
\end{tabular} \\
\hline Carroll, 2001 & & $\begin{array}{l}\text { 1) O estudo avaliou indivíduos fora da faixa etária } \\
\text { de adultos idosos; } \\
\text { 2) Não foi determinado o período do estudo; } \\
\text { 3) Não houve randomização. }\end{array}$ & $\begin{array}{l}\text { O treinamento de resistência promove alterações } \\
\text { neurológicas funcionais e adaptativas, afetando a } \\
\text { coordenação sensorial e motora. }\end{array}$ \\
\hline
\end{tabular}


Conclusão

\begin{tabular}{|c|c|c|c|}
\hline $\begin{array}{c}\text { Detalhes do } \\
\text { estudo }\end{array}$ & Detalhes da intervenção & \multirow{2}{*}{ Fragilidade/viés do estudo } & \multirow{2}{*}{ Resultados } \\
\hline Autor, ano & Duração (semanas) & & \\
\hline Kim, 2010 & 8 & & $\begin{array}{l}\text { O exercício físico promoveu uma redução } \\
\text { satisfatória do risco de quedas entre adultos idosos. }\end{array}$ \\
\hline Taguchi, 2010 & 52 & 1) Não houve randomização. & $\begin{array}{l}\text { Um longo programa de exercícios variados pode } \\
\text { melhorar ou manter performance física de idosos } \\
\text { saudáveis. }\end{array}$ \\
\hline Peterson, 2011 & & $\begin{array}{l}\text { 1) Eventuais vieses de publicação dos estudos } \\
\text { analisados. }\end{array}$ & $\begin{array}{l}\text { O exercício resistido promove aumento de massa } \\
\text { muscular entre adultos idosos. }\end{array}$ \\
\hline $\begin{array}{l}\text { Drummond, } \\
2008\end{array}$ & & $\begin{array}{l}\text { 1) Indivíduos de diferentes faixas etárias; } \\
\text { 2) Não houve randomização. }\end{array}$ & $\begin{array}{l}\text { A estimulação da síntese proteica muscular } \\
\text { é atrasada em homens idosos, a despeito do } \\
\text { exercício resistido e da suplementação com } \\
\text { aminoácidos. }\end{array}$ \\
\hline
\end{tabular}

Fonte: elaborado pelos autores.

\section{DISCUSSÃO}

O presente trabalho está assentado em evidências de ensaios clínicos, estudos com grupo controle, metanálises, estudo longitudinal, revisões de literatura. O primeiro viés dessa construção seria a falta de experimentação dos trabalhos de revisão. Contudo, é preciso salientar que eles levantam discussões cabíveis e norteadoras, propondo pontos relevantes a serem discutidos no que se refere ao tema ora discorrido.

Os demais trabalhos, conforme o que já foi exposto, testaram variáveis que, por vezes, coincidem. Os grupos de indivíduos avaliados, em sua quase totalidade, envolviam pessoas idosas.

O tipo de exercício foi avaliado quanto a sua capacidade de induzir algum aumento na densidade mineral óssea. Duas abordagens de exercício foram comparadas em três grupos de mulheres idosas menopausadas: exercício funcional (duas vezes por semana) e exercício resistido (uma e duas vezes por semana). Não houve diferença significativa entre esses grupos ao longo de um ano de estudo. Sugere-se que, possivelmente, o tempo médio de treino ou a interrupção dos treinos, dependendo da estação do ano, poderiam ser fatores limitantes para o aumento da matriz óssea. Outra explicação plausível seria a incapacidade dessas mulheres atingirem um limiar de força necessária à síntese de substrato para o córtex ósseo pelos osteoblastos. ${ }^{7}$ Ainda discorrendo sobre saúde osteomuscular de mulheres adultas idosas, há uma hipótese de que o exercício resistido a longo prazo poderia retardar a progressão da artrite, avaliando-se através de ressonância magnética a articulação do joelho, ${ }^{8}$ bem como em 106 mulheres menopausadas, tratadas e sobreviventes do câncer de mama com mais de 50 anos divididas em dois grupos: exercício resistido + exercício de impacto e exercício de flexibilidade, foi visto que aquelas que se submeteram ao exercício resistido + exercício de impacto e faziam uso de inibidores da aromatase obtiveram um aumento de massa muscular com melhora no equilíbrio, redução do risco de fraturas, do risco de quedas e mantiveram a densidade óssea semelhante ao início do estudo, ou seja, houve evidência de que o exercício resistido nessa amostra preveniu acerca da perda de massa óssea. ${ }^{9}$ Um outro aspecto que envolveu a comparação entre essas modalidades de exercício mencionadas anteriormente foi a capacidade de melhorar o equilíbrio em idosos. Foi visto que, em apenas 6 meses, observando indivíduos com idade média de 65 anos, o grupo submetido a exercícios resistidos 4 vezes por semana se sobressaiu em relação àquele submetido ao exercício funcional. Acredita-se que, ao serem trabalhados determinados grupamentos musculares, seria possível regular o centro de massa e corrigir a postura, além de estimular fibras musculares e aumentar sua força contrátil, o que, por sua vez, melhoraria o equilíbrio e preveniria contra a possibilidade de quedas. ${ }^{10}$ Adaptações neurais devem ocorrer em indivíduos jovens que foram submetidos ao longo de um determinado período a um exercício resistido, melhorando a capacidade de coordenação através de estimulação das fibras musculares e vias neuronais específicas, o que poderia ocorrer, também, em idosos e explicaria modificações na marcha e no equilíbrio. ${ }^{15}$ É possível que este raciocínio encontre mais evidências em um ensaio avaliando a melhora da capacidade física e cognitiva (através de avaliação neuropsicológica e dosagem sérica do fator neurotrófico derivado cerebral, Brain-derived Neurotrophic Factor (BDNF)) de 100 mulheres entre 65-75 anos submetidas a uma série variada de exercícios físicos duas vezes por semana durante 60 minutos, envolvendo exercício aeróbico, resistido, de equilíbrio, flexibilidade, coordenação e agilidade. ${ }^{11}$

Um grande tema a ser discutido seria a capacidade de ganho de força atribuída ao exercício resistido realizado por indivíduos idosos. Evidências mostram, com significância relevante, que é possível promover ganho de força, avaliandose o desempenho dos membros superiores e inferiores, quando submetidos ao exercício resistido, avaliando-se indivíduos submetidos a exercícios de agachamento, cadeira extensora, supino $90^{\circ}$ e remada, por exemplo. Haveria, também, a possibilidade de implementar o ganho de força através do aumento de intensidade de baixa, moderada à alta. Outra variável a ser avaliada seria a diferença entre gênero e idade quanto à capacidade de ganho de força e, ao que indicam as evidências, parece haver uma similaridade entre homens 
e mulheres; e adultos jovens e adultos idosos. Entretanto, acredita-se que a atividade física possa apenas retardar o declínio da performance muscular. É preciso considerar doenças de base, o grau de funcionalidade prévio dos indivíduos e os cuidados com a saúde prévios (alimentação e hábitos, principalmente), a fim de se possa estabelecer uma relação de causa de efeito com relação ao ganho de força muscular e o exercício resistido. ${ }^{1} \mathrm{~A}$ importância do exercício resistido em determinados grupamentos musculares encontra evidência na avaliação de um estudo com grupo controle, envolvendo dezoito idosos voluntários, divididos em três grupos de seis, sendo um grupo controle e os demais grupos de intervenção (exercício resistido e exercício de equilíbrio), avaliados durante oito semanas. Viu-se que aqueles submetidos ao exercício resistido obtiveram melhora na marcha e no equilíbrio em relação aos demais grupos, reduzindo o risco de quedas por "derrapagem", provavelmente, devido ao ganho de torque pela hipertrofia dos músculos extensores da perna, o que favoreceria uma caminhada com um momento força adequado com menos tempo de contato com o calcanhar, suprimindo, quiçá, a perda de força oriunda da sarcopenia senil. ${ }^{16}$ No Japão, 33 idosos realizaram séries de exercícios aeróbicos e anaeróbicos durante doze meses e também conseguiram bons resultados quanto à hipertrofia dos membros inferiores, equilíbrio e marcha, e teriam revertido, em parte, as pequenas incapacidades funcionais desses indivíduos, reforçando a hipótese de que o exercício poderia configurar uma futura abordagem de prevenção da perda funcional osteomuscular em idosos, uma vez que é de baixo custo e fácil reprodução, contribuindo para a redução da mortalidade e da morbidade (diminuição da necessidade de cuidados a longo prazo) nessa população. Era esperado um implemento da qualidade de vida a partir do início da prática de exercício, entretanto, tal fato não pode ser verificado nesse estudo realizado no Japão por falta de um tempo e de uma quantidade maior de indivíduos para melhorar a análise estatística. ${ }^{17}$ Talvez, o ganho de força possa ser observado medindo-se a massa musculoesquelética per si, que poderia aumentar em indivíduos submetidos, em média, a cinco semanas de exercício resistido. Essa evidência poderia justificar a necessidade de exercício naqueles indivíduos por volta da $6^{\mathrm{a}}$ década de vida, que costumam apresentar perda de equilíbrio, alterações na marcha, mobilidade e dificuldade de realizar atividades cotidianas (fazer compras, limpar a casa, etc.). Apesar disso, a mensuração da massa musculoesquelética não forneceria detalhes como mudanças na morfologia, volume, comprimento dos sarcômeros, que seriam parâmetros importantes para atestar microscopicamente o implemento funcional das fibras musculares. Todavia, a verificação da massa musculoesquelética, comparando-se jovens adultos ao longo do tempo, bem como comparando-os com idosos ao mesmo tempo, mostrou que, possivelmente, a sarcopenia seria prevenida ou retardada naqueles que, precocemente, começaram a se submeter ao exercício resistido e, ainda, até os mais idosos, conseguiriam obter um ganho de massa musculoesquelética, quando submetidos por um determinado tempo ao exercício resistido. ${ }^{18} \mathrm{Em}$ idosos obesos, avaliandose dois grupos: um submetido a uma dieta com restrição calórica e mudança de hábitos e outro submetido a dieta e exercício resistido, foi visto uma menor redução no percentual de gordura corporal e uma menor perda de peso naqueles que realizavam exercício. Em contrapartida, houve aumento de força, indicando ganho de massa musculoesquelética naqueles se exercitavam. ${ }^{12}$ De forma semelhante, o fato observado anteriormente foi reproduzido em um ensaio com 93 indivíduos divididos em três grupos: 1) dieta; 2) exercício resistido; 3) dieta + exercício resistido. Similarmente ao que Frimel e seus colaboradores tentaram demonstrar, houve aumento de força física com preservação de massa muscular e densidade mineral óssea, bem como melhora no equilíbrio e na marcha, no grupo exercício resistido + dieta. No entanto, se comparado ao grupo submetido somente à dieta, o grupo exercício + dieta obteve menor perda de peso, porém reverteram a sarcopenia, reduzindo a sua fragilidade, incapacidade e melhoraram quanto à sua independência para realizar atividades diárias, o que justificaria a implementação de um programa envolvendo dieta e exercício físico para idosos obesos como forma de prevenção e redução de gastos em saúde pública ${ }^{13}$. Avaliandose indivíduos com elevado teor de gordura intermuscular e com alto índice de massa corpórea, foi visto que não há diferença quanto ao tipo de exercício realizado, se o exercício resistido tradicional ou o excêntrico. Todavia, indivíduos com alto percentual de gordura intermuscular perderam mais massa muscular do que aqueles com menor percentual, mas haveria a possibilidade desse fato ser explicado por conta de um viés do screening dos indivíduos no grupo com alto percentual de gordura intermuscular conter um maior número de indivíduos com diabetes mellitus. ${ }^{2}$

Uma boa nutrição seria importante para um bom desempenho muscular, quando um indivíduo é submetido ao exercício físico. Logo, assim como os jovens, os idosos também necessitariam de um bom aporte nutricional, envolvendo, principalmente, aminoácidos essenciais para estimular a síntese proteica musculoesquelética. Apesar disso, parece haver um retardo na sinalização via quinases (Extracellular Signal-Regulated Kinases (ERK), AMP-Activated Protein Kinase (AMPK) e Mechanistic Target of Rapamycin(mTOR), principalmente) para o início da síntese de proteínas após a realização de exercício resistido. Sugere-se que esse atraso ocorreria em virtude de uma dessensibilização para a secreção insulínica em indivíduos idosos ou, devido a uma perda de massa muscular em geral e uma perda, especificamente, de fibras musculares do tipo2, implicariam um menor grau de tensão durante o exercício e uma menor produção de lactato (representando um grande estresse celular se comparado aos jovens), o que poderia interferir na ativação das quinases sinalizadoras da síntese proteica. ${ }^{18}$ Além disso, um outro aspecto que poderia interferir na síntese proteica seria o uso de analgésicos, algo muito comum em idosos. Foi avaliado o uso de acetominofeno (Paracetamol) na dose $1000 \mathrm{mg} /$ dia em comparação ao placebo em idosos submetidos, durante 4 meses, ao exercício resistido. Os indivíduos que tomavam de fato o acetominofeno obtiveram um aumento de força e de massa muscular superior ao placebo. Tal fato poderia ser explicado pela inibição das prostaglandinas, promovendo um maior efeito anabólico em detrimento do efeito catabólico. ${ }^{14}$ 
Por caracterizar-se como uma revisão sistemática, o presente estudo possui as limitações inerentes a pesquisa observacional retrospectiva, ao compartilhar dos potenciais de vieses e falhas dos artigos em que se baseou.

\section{CONCLUSÃO}

Acreditamos que os temas sobre os quais discorremos ainda carecem de mais evidências experimentais. Contudo, os estudos, até o momento, indicam que a atividade física através do exercício resistido seria benéfica para indivíduos idosos que padecem com as alterações patológicas associadas ao envelhecimento do sistema osteomuscular (sarcopenia,

\section{REFERÊNCIAS}

1. Peterson MD, Rhea MR, Sen A, Gordon PM. Resistance exercise for muscular strength in older adults: A meta-analysis. Ageing Res Rev. 2010;9(3):226-37.

2. Jacobs JL, Marcus RL, Morrell G, LaStayo P. Resistance exercise with older fallers: its impact on intermuscular adipose tissue. Biomed Res Int. 2014;(2014):1-7.

3. Sundell, J. Resistance training is an effective tool against metabolic and frailty syndromes. Adv Prev Med. 2011;(2011):1-7.

4. Cruz-Jentoft AJ, Baeyens JP, Bauer JM, Boirie Y, Cederholm T, Landi F, et al. Sarcopenia: European consensus on definition and diagnosis: Report of the European Working Group on Sarcopenia in Older People. Age Ageing. 2010;39(4):412-23.

5. Gordon PM, Liu D, Sartor MA, IglayReger HB, Pistilli EE, Gutmann L, et al. Resistance exercise training influences skeletal muscle immune activation: a microarray analysis J Appl Physiol. 2012;112(3):443-53.

6. Rutherford OM. Is there a role for exercise in the prevention of osteoporotic fractures? Br J Sports Med. 1999;33(6):378-86.

7. Ashe MC, Gorman E, Khan KM, Brasher PM, Cooper DM, McKay HA, et al. Does frequency of resistance training affect tibial cortical bone density in older women? A randomized controlled trial. Osteoporos Int. 2013;24(2):623-32.

8. Lange AK, Vanwanseele B, Foroughi N, Baker MK, Shnier R, Smith RM, et al. Resistive Exercise for Arthritic Cartilage Health (REACH): a randomized double-blind, sham-exercise controlled trial. BMC Geriatr. 2009;9:1.

9. Winters-Stone KM, Dobek J, Nail L, Bennett JA, Leo MC, Naik A, et al. Strength training stops bone loss and builds muscle in postmenopausal breast cancer survivors: a randomized, controlled trial. Breast Cancer Res Treat. 2011;127(2):447-56.

10. Joshua AM, D’Souza V, Unnikrishnan B, Mithra P, Kamath A, Acharya $\mathrm{V}$, et al. Effectiveness of progressive resistance strength training versus traditional balance exercise in improving balance among the elderly - a randomised controlled trial. J Clin Diagn Res. 2014;8(3):98-102. osteoporose e artrose). Admitimos que, independentemente da idade de início das atividades, do subgrupo populacional ou das variáveis clínicas e funcionais apresentadas, haveria um ganho, a longo prazo, em termos de saúde pública. Haveria redução do risco associado a quedas, melhoria na qualidade de vida, na expectativa de vida livre de limitações e maior liberdade e independência na execução das atividades da vida diária. Poderíamos estabelecer a prática do exercício resistido para a população idosa como parte de uma "terapia", pois há um saldo positivo de evidências, mesmo que os diversos programas de exercícios ainda estejam em construção através de ensaios para definir a melhor forma de realização.

11. Vaughan S, Morris N, Shum D, O'Dwyer S, Polit D. Study protocol: a randomised controlled trial of the effects of a multi-modal exercise program on cognition and physical functioning in older women. BMC Geriatr. 2012;12:60.

12. Frimel TN, Sinacore DR, Villareal DT. Exercise attenuates the weight-loss-induced reduction in muscle mass in frail obese older adults. Med Sci Sports Exerc. 2008;40(7):1213-9.

13. Villareal DT, Chode S, Parimi N, Sinacore DR, Hilton T, Armamento-Villareal R, et al. Weight loss, exercise, or both and physical function in obese older adults. $\mathrm{N}$ Engl $\mathrm{J}$ Med. 2011;364(13):1218-29.

14. Jankowski CM, Gozansky WS, MacLean PS, Shulman B, Wolfe P, Schwartz RS, et al. N-acetyl-4-aminophenol and musculoskeletal adaptations to resistance exercise training. Eur J Appl Physiol. 2013;113(5):1127-36.

15. Carroll TJ, Barry B, Riek S, Carson RG. Resistance training enhances the stability of sensorimotor coordination. Proc Biol Sci. 2001;268(1464):221-7.

16. Kim S, Lockhart T. Effects of 8 weeks of balance or weight training for the independently living elderly on the outcomes of induced slips. Int J Rehabil Res. 2010;33(1):49-55.

17. Taguchi N, Higaki Y, Inoue S, Kimura H, Tanaka K. Effects of a 12-month multicomponent exercise program on physical performance, daily physical activity, and quality of life in very elderly people with minor disabilities: an intervention study. J Epidemiol. 2010;20(1):21-9.

18. Peterson MD, Sen A, Gordon PM. Influence of resistance exercise on lean body mass in aging adults: a meta-analysis. Med Sci Sports Exerc. 2011;43(2):249-58.

19. Drummond MJ, Dreyer HC, Pennings B, Fry CS, Dhanani S, Dillon EL, et al. Skeletal muscle protein anabolic response to resistance exercise and essential amino acids is delayed with aging. J Appl Physiol. 2008;104(5):1452-61.

\section{Como citar:}

Rego LA, Patriota MB Filho, Cavalcante JC, Linhares JP, Leite JA. Efeito musculoesquelético do exercício resistido em idosos: revisão sistemática. Rev Med UFC. 2016 jul-dez;56(2):39-46. 